\title{
Knowledge and attitude towards basic life support among primary health care physicians in Taif, Saudi Arabia, in 2018: a regression analysis
}

\author{
Mohammed Mihmas Albaqami ${ }^{1}$, Hani Abozaid ${ }^{2}$
}

${ }^{1}$ Saudi Board of Family Medicine, Ministry of Health, Taif, Kingdom of Saudi Arabia

${ }^{2}$ Associate Professor, Family and Community Medicine Department, Faculty of Medicine, Taif University, Taif, Kingdom of Saudi Arabia

Type of article: Original

\begin{abstract}
Background: Relatively few studies have been carried out regarding assessment of knowledge and attitude of primary care physicians in Saudi Arabia towards Basic Life Support (BLS) and its training, as most of the studies were carried out among health care professionals in general, with the emphasis on hospital workers.

Aim: The aim of the study was to determine the knowledge and attitude of primary health care physicians in Taif towards BLS, in order to identify deficient points for further intervention.

Methods: This cross-sectional study was conducted in a random sample of primary health care centers in Taif city, Saudi Arabia, from January 2018 to June 2018. Primary health care physicians working in these centers constituted the target population of the study. A pre-designed self-administered questionnaire was used to collect data from the physicians. The questionnaire included sociodemographic data, history of attending training courses in BLS and obtaining a BLS license, and knowledge and attitude. Data were analyzed by IBM-SPSS version 22, using descriptive statistics, Chi-square test of independence, multivariate logistic regression, and calculating the odds ratios and adjusted odds ratios. A p-value of $\leq 0.05$ was considered statistically significant.

Results: One hundred and eighty three primary health care physicians were included in the study. About twothirds of them (65.6\%) were males. The majority of the participants $(82 \%)$ were non-Saudis. All participants had attended BLS training courses. The majority of them (98.9\%) had a valid BLS certificate. Overall, satisfactory knowledge regarding BLS was observed among 56.3\% of the PHC physicians. Multivariate logistic regression analysis revealed that non-Saudi physicians had a lower probability of having unsatisfactory BLS knowledge compared to Saudi physicians (Adjusted odds ratio "AOR" $=0.30,95 \%$ CI: $0.12-0.75, p=0.010$ ). Compared to physicians with 5 years or less experience in PHC practice, those having more than 10 years of practice were at almost three-fold the risk of having unsatisfactory BLS knowledge ( $\mathrm{AOR}=2.99,95 \% \mathrm{CI}: 1.27-7.03, \mathrm{p}=0.012$ ). Considering physicians who had Bachelor of Medicine (MBBS) as a reference category, Master holder physicians were less likely to have unsatisfactory BLS knowledge (AOR $=0.17,95 \%$ CI:0.03-0.83, $\mathrm{p}=0.030$ ), whereas FM board certified physicians were more likely to have unsatisfactory BLS knowledge (AOR=5.14, 95\% CI: 2.28-21.08, $\mathrm{p}=0.004)$. Physicians`age was significantly associated with BLS knowledge ( $\mathrm{p}=0.017)$, but the magnitude of the association was low (Cramer's V coefficient $=0.211$ ).

Conclusion: Knowledge of physicians regarding BLS was unsatisfactory in some issues. More than half of the PHC physicians had satisfactory knowledge. Attitude of the physicians towards BLS was positive in Saudi physicians, and family medicine residents had more negative attitude towards BLS than their counterparts did. Since the BLS certificate is validated for two years, additional courses could be given to primary health care physicians between the mandatory courses.
\end{abstract}

Keywords: Basic Life Support, Cardio-Pulmonary Resuscitation, American Heart Association

\section{Corresponding author:}

Mohammed Mihmas Albaqami, Saudi Board of Family Medicine, Ministry of Health, Taif, Kingdom of Saudi Arabia. Tel: Telephone: +966-500990321, Email: mido.22@windowslive.com

Received: September 12, 2018, Accepted: November 18, 2018, Published: Match 2019

iThenticate screening: November 15, 2018, English editing: November 26, 2018, Quality control: January 06, 2019

This article has been reviewed / commented by three experts

Ethics approval: (Ref: 51, date: January 10, 2018, regional Research and Ethical Committee, Taif)

(C) 2019 The Authors. This is an open access article under the terms of the Creative Commons Attribution-NonCommercialNoDerivs License, which permits use and distribution in any medium, provided the original work is properly cited, the use is non-commercial and no modifications or adaptations are made. 


\section{Introduction}

Basic Cardiac Life Support (BLS) is essential and can save lives, especially among health care providers who encounter ill patients every day during their practice (1). In 1984, the American Heart Association (AHA) introduced Cardiopulmonary resuscitation (CPR) into Saudi Arabia (2). Currently, the Saudi Heart Association (SHA) is responsible for conducting BLS training courses (2). The Basic Cardiac Life Support (BLS) course is now mandatory for all health care providers for their practice. The course is held in standard centers approved by the health authorities. BLS is important for saving lives after cardiac arrest. Essential steps of BLS include immediate recognition of sudden cardiac arrest, activation of the emergency response system, early CPR, and rapid defibrillation with an automated external defibrillator (AED) (3). Lack of BLS training and negative attitude towards it leads to inability of PHC physicians to cope with medical emergencies at their workplace and can lead to adverse consequences. We do not know the actual knowledge and the attitude of the PHC physicians in Taif toward the BLS courses. To the best of our knowledge, no one has explored the knowledge and attitude of PHC physicians in Taif city towards BLS training. The general objective of this research was to determine the knowledge and attitude of PHC physicians in Taif towards BLS in order to identify deficient points for further intervention. The specific objectives were to estimate the percentage of PHC physicians in Taif who have a valid BLS certificate; and to determine the knowledge and attitudes of PHC physicians in Taif regarding BLS.

\section{Material and Methods}

\subsection{Research design and setting}

This cross-sectional study was implemented on primary health care physicians working at PHC centers for the Ministry of Health (MOH) inside and outside Taif city from January 2018 to June 2018. Taif city is located in the West of Saudi Arabia. The estimated population is 1,281,613 according to the 2011 census (4). In Taif, there are 121 PHC centers belonging to the Ministry of Health: 19 inside Taif city and 102 outside Taif city. They include all 350 physicians. This study was conducted in a random sample of these primary health care centers.

\subsection{Sample size}

The required sample size was estimated using the online sample size calculator (5) using this formula: $\mathrm{n}=\mathrm{P}(1-\mathrm{P})(\mathrm{Z} / \mathrm{E})^{2}$, where $\mathrm{Z}=$ the value from the standard normal distribution reflecting the confidence level of $95 \%$ (1.96), $\mathrm{E}=$ the desired margin of error $(0.05), \mathrm{P}=$ the proportion of $\mathrm{BF}$ in the population $(\mathrm{p}=0.5$ i.e. $50 \%$ was used to generate the largest sample size). Assuming that the confidence interval as $5 \%$, at $95 \%$ confidence level, the required sample size out of 350 physicians was 183 physicians $(52.3 \%)$.

\subsection{Instrument}

A pre-designed self-administered questionnaire was used to collect data from the physicians. The questionnaire included:

A) Personal data: gender, nationality, age, working sector, educational level, experience in PHC practice.

B) History of attending training courses in BLS and having a BLS license.

C) Knowledge aspect: determined by fifteen multiple-choice questions based on the recent American Heart Association guidelines (6).

D) Attitude aspect: determined by 4 questions updated from a previous study (7). A five-point Likert scale ranging between strongly agree to strongly disagree was used.

Face validity of the questionnaire was ascertained by four consultants in the fields of Family Medicine, Community Medicine, Emergency Medicine, and instructor of BLS training courses.

\subsection{Pilot study}

The pilot study was carried out by twenty physicians who tested the understanding of the research questions and estimated the time needed to complete it. Their results were added in the final report since there was no difference from the main results. Cronbach's alpha $=0.788$

\subsection{Data collection}

The main researcher visited the physicians in their offices to distribute and collect the data in person and was available during the distribution of the questionnaire for any clarification. All the physicians participated in the research. Physicians` knowledge about BLS was calculated as follows: The physicians were asked to respond to questions about BLS. The correct answers were given a score of " 1 " while the wrong or missing answers were given a score of "0". The overall score was calculated for every physician. The mean value of the overall score was utilized as a cut-off point for knowledge categorization. Physicians were classified as having satisfactory 
knowledge, if they had an overall score greater than or equal to the mean score, and as having unsatisfactory knowledge if their score was less than the mean score. Responses of the physicians to attitude statements were scored in a way that the higher the score, the more positive the attitude toward BLS training. The median value of the total score was utilized as a cut-off point for knowledge categorization. Physicians were classified as having positive attitude, if they had an overall score $\geqslant$ the mean score and as having negative attitude, if their score was less the mean score.

\subsection{Data analysis}

The data were collected and verified by hand then coded before entry. Statistical Package for Social Sciences IBMC SPSS $\odot$ Statistics version 22 (IBM $\odot$ Corp., Armonk, NY, USA) was used for data entry and analysis. Descriptive statistics (e.g. frequencies and percentage) and analytic statistics were applied for comparison using Chi-square test of independence, and Multivariate Logistic Regression with its adjusted Odds ratio and 95\% confidence interval. Pvalue less than 0.05 was considered statistically significant.

\subsection{Ethical consideration}

The Declaration of Helsinki - ethical principles for medical research involving human subjects (adopted by the 18th General Assembly of World Medical Association (WMA), Helsinki, Finland, June 1964 and last amended by the 64th WMA General Assembly, Fortaleza, Brazil, October 2013) was considered throughout the designing of the study protocol and its implementation. In detail:

1) Permission from the regional Research and Ethical Committee in Taif was granted for conducting the study (Ref: 51, date: January 10, 2018).

2) Permission from the source of our adapted questionnaire was obtained.

3) All the information in the questionnaires was kept confidential.

4) The participants were provided with an information sheet that included information about the topic and aim of the study, its procedure, and other relevant information. Written informed consent was obtained from formal participants before participating in the study.

5) All the subjects participated voluntarily in the study.

\section{Results}

\subsection{Sociodemographic and general findings}

One hundred eighty-three primary health care physicians were included in the study. Table 1 presents their personal characteristics. About two-thirds of them (65.6\%) were males and the age of $44.3 \%$ of them ranged between 31 and 40 years whereas that of $28.4 \%$ ranged between 25 and 30 years. The majority of the participants $(82 \%)$ were nonSaudis. Most of them (70.5\%) had MBBS qualification whereas 13.1\% were Family Medicine Board certified. The experience in PHC was 5 years or less among $41.5 \%$ of the physicians while it exceeded 10 years among $28.4 \%$ of them. All participants had attended a BLS training course. The majority of them $(98.9 \%)$ had a valid BLS certificate.

Table 1. Personal characteristics of the participated primary health care physicians $(\mathrm{n}=183)$.

\begin{tabular}{|l|l|l|l|}
\hline Variables & Categories & $\mathrm{n}$ & $\%$ \\
\hline \multirow{4}{*}{ Age (year) } & $25-30$ & 52 & 28.4 \\
\cline { 2 - 4 } & $31-40$ & 81 & 44.3 \\
\cline { 2 - 4 } & $>40$ & 50 & 27.3 \\
\hline \multirow{3}{*}{ Nender } & Mate & 120 & 65.6 \\
\cline { 2 - 4 } & Female & 63 & 34.4 \\
\hline \multirow{5}{*}{ Educationality level } & Saudi & 33 & 18.0 \\
\cline { 2 - 4 } & Non-Saudi & 150 & 82.0 \\
\hline \multirow{5}{*}{ Experience in PHC practice (year) } & MBBS & 129 & 70.5 \\
\cline { 2 - 4 } & Diploma & 15 & 8.2 \\
\cline { 2 - 4 } & Master & 15 & 8.2 \\
\cline { 2 - 4 } & Family Medicine Board & 24 & 13.1 \\
\cline { 2 - 4 } & $\mathbf{6 - 1 0}$ & 76 & 41.5 \\
\cline { 2 - 4 } & $>10$ & 55 & 30.1 \\
\hline & & 52 & 28.4 \\
\hline
\end{tabular}




\subsection{BLS Knowledge}

\subsubsection{Descriptive findings}

The majority of the physicians could recognize that "Call emergency, CPR, Defibrillation, Advanced care" is the correct sequence for the chain of survival (95.6\%). The critical characteristics of high-quality CPR include starting chest compressions within 10 seconds of recognition of cardiac arrest, pushing hard and fast and minimizing interruptions (94\%), the technique to open the airway of an unconscious casualty is head tilt and chin lift (91.3), the signs of airway obstruction are poor air exchange, high-pitched noise while inhaling and inability to speak (90.2\%) and the correct ratio of chest compressions to rescue breaths for use in CPR of an adult casualty is 30 compressions: 2 rescue breaths $(83.6 \%)$. Only $53 \%$ of the physicians knew that the 2015 AHA guidelines for CPR recommended BLS sequence of steps are Chest compressions, Airway, and Breathing. Less than half of the participants knew correctly the five steps in the Adult Chain of Survival (44.3\%). The total knowledge score ranged between 3 and 15 with a median value of 11 . Overall, satisfactory knowledge regarding BLS was observed among $56.3 \%$ of the PHC physicians as illustrated.

\subsubsection{Univariate statistical analysis}

The association between physicians' age and BLS knowledge was statistically significant $(p=0.017)$, but the magnitude of the association was low (Cramer's V coefficient $=0.211$ ). There was no significant association between physicians' gender and level of knowledge regarding BLS. Regarding the physicians' nationality, $62 \%$ of non-Saudi physicians compared to only $30.3 \%$ of Saudi physicians had a satisfactory BLS knowledge level, which had significant difference $(\mathrm{p}=0.001)$ but low correlation was evident (Cramer's V coefficient $=0.246)$. Regarding the physicians`educational level, the association between physicians` educational level and BLS knowledge level was statistically significant $(\mathrm{p}<0.001$, Cramer's V Coefficient $=0.317)$. Also, the study showed significant association between physicians`experience in PHC practice and BLS knowledge ( $\mathrm{p}=0.031)$ (Table 2).

Table 2. Association between physicians' socio-demographic characteristics, and their attitudes and knowledge regarding BLS.

\begin{tabular}{|l|l|l|l|l|}
\hline \multirow{2}{*}{ Variables } & \multicolumn{2}{l|}{ Knowledge } & \multicolumn{2}{l|}{ Attitudes } \\
\cline { 2 - 5 } & $\mathrm{X}^{2}$ & $\mathrm{p}$-value & $\mathrm{X}^{2}$ & $\mathrm{p}$-value \\
\hline Age & 8.16 & 0.017 & 6.89 & 0.032 \\
\hline Gender & 0.02 & 0.886 & 0.80 & 0.372 \\
\hline Nationality & 11.05 & 0.001 & 8.54 & 0.003 \\
\hline Education level & 18.33 & $<0.001$ & 13.88 & 0.003 \\
\hline Experience in PHC practice (years) & 6.97 & 0.031 & 3.23 & 0.199 \\
\hline Having valid BLS certificate & -- & $0.190^{*}$ & -- & $0.167^{*}$ \\
\hline
\end{tabular}

* Fischer exact test

\subsubsection{Multivariate logistic regression analysis}

Table 3 reveals that non-Saudi physicians had lower probability of having unsatisfactory BLS knowledge compared to Saudi physicians (Adjusted odds ratio "AOR" $=0.30,95 \%$ CI: $0.12-0.75, \mathrm{p}=0.010$ ). Compared to physicians with 5 years or less experience in PHC practice, those having more than 10 years of practice were at almost three-fold the risk of having unsatisfactory BLS knowledge $(\mathrm{AOR}=2.99,95 \% \mathrm{CI}: 1.27-7.03, \mathrm{p}=0.012)$. Considering physicians who had MBBS as a reference category, Master holder physicians were less likely to have unsatisfactory BLS knowledge $(\mathrm{AOR}=0.17,95 \% \mathrm{CI}: 0.03-0.83, \mathrm{p}=0.030$ ) whereas $\mathrm{FM}$ board certified physicians were more likely to have unsatisfactory BLS knowledge $(\mathrm{AOR}=5.14,95 \% \mathrm{CI}: 2.28-21.08, \mathrm{p}=0.004)$. Physicians' age was not significantly associated with BLS knowledge.

\subsection{Attitude towards BLS training}

\subsubsection{Descriptive findings}

All the primary health care physicians thought that BLS training should be mandatory. The majority of them are interested in gaining more skills and knowledge of BLS (97.3\%), willing to gain additional BLS knowledge in the form of a BLS course $(96.2 \%)$ and either strongly agreed or agreed that BLS courses should be free for PHC physicians $(96.7 \%)$. Overall, positive attitude towards BLS training was observed among almost two-thirds of the participants $(65.5 \%)$ (Table 2).

\subsubsection{Univariate statistical analysis}

The study showed significant association between physicians' age and attitude towards BLS training ( $\mathrm{p}=0.032$ ). There was no statistically significant association between physicians' gender and attitude towards BLS training, but there was significant association between Physicians` nationality and their attitude towards BLS training $(\mathrm{p}=0.003)$. 
The association between physicians` educational level and attitude towards BLS training was statistically significant $(\mathrm{p}=0.003)$, but there was no significant association between physicians' experience in PHC practice and attitude towards BLS training. Likewise, the association between having a valid BLS certificate and attitude towards BLS training was not significant. Finally, the study showed that $35.2 \%$ of physicians who had positive attitude regarding BLS training compared to $56 \%$ of those who had negative attitude expressed satisfactory knowledge regarding BLS, which suggests a significant association between attitude towards BLS training and knowledge about it $(\mathrm{p}=0.005)$.

3.3.3. Multivariate logistic regression analysis

Table 3 reveals that non-Saudi physicians were more likely to have positive attitude towards BLS training compared to Saudi physicians $(\mathrm{AOR}=2.18,95 \% \mathrm{CI}: 1.01-5.05, \mathrm{p}=0.050)$. Considering physicians who had MBBS as a reference category, FM board certified physicians were less likely to have positive attitude towards BLS training (AOR $=0.52,95 \%$ CI: $0.09-0.69, \mathrm{p}=0.007$ ). Physicians` age was not significantly associated with attitude towards BLS training.

Table 3. Predictors of having unsatisfactory BLS knowledge among primary health care physicians in Taif (Multivariate logistic regression analysis)

\begin{tabular}{|c|c|c|c|c|c|c|c|}
\hline \multicolumn{3}{|l|}{ Variables } & B & SE & Adjusted OR & $95 \% \mathrm{CI}$ & $\mathrm{p}$-value \\
\hline \multirow{9}{*}{$\begin{array}{l}\text { having unsatisfactory BLS } \\
\text { knowledge }\end{array}$} & \multirow[t]{2}{*}{ Nationality } & Saudi & & & --- & --- & \\
\hline & & Non-Saudi & -1.214 & 0.474 & 0.30 & $0.75-0.12$ & 0.010 \\
\hline & \multirow{3}{*}{$\begin{array}{l}\text { Experience in } \\
\text { PHC practice } \\
\text { (years) }\end{array}$} & $\leq 5 *$ & & & --- & --- & \\
\hline & & $10-6$ & -0.268 & 0.420 & 0.77 & $1.74-0.34$ & 0.523 \\
\hline & & $10<$ & 1.096 & 0.436 & 2.99 & $7.03-1.27$ & 0.012 \\
\hline & \multirow[t]{4}{*}{ Educational level } & MBBS a & & & --- & --- & \\
\hline & & Diploma & 0.042 & 0.591 & 1.04 & $3.32-0.33$ & 0.943 \\
\hline & & Master & -1.779 & 0.817 & 0.17 & $0.84-0.03$ & 0.030 \\
\hline & & Family Medicine Board & 1.637 & 0.567 & 5.14 & $21.08-2.28$ & 0.004 \\
\hline \multirow{6}{*}{$\begin{array}{l}\text { Having positive attitude } \\
\text { toward BLS training }\end{array}$} & \multirow[t]{2}{*}{ Nationality } & Saudi & & & --- & --- & \\
\hline & & Non-Saudi & 0.778 & 0.429 & 2.18 & $5.05-1.01$ & 0.050 \\
\hline & \multirow[t]{4}{*}{ Educational level } & MBBS a & & & & --- & \\
\hline & & Diploma & 0.099 & 0.582 & 1.10 & $3.45-0.35$ & 0.865 \\
\hline & & Master & 0.361 & 0.616 & 1.43 & $4.80 \_0.43$ & 0.558 \\
\hline & & Family Medicine Board & -1.398 & 0.522 & 0.25 & $0.69 \_0.09$ & 0.007 \\
\hline
\end{tabular}

Age was removed from the final logistic regression model (not significant). * Reference category, B: Slope, SE: Standard error, OR: Odds ratio, CI: Confidence interval.

\section{Discussion}

According to AHA (2015) BLS guidelines, immediate recognition of cardiac arrest, early activation of emergency medical services (EMS), early CPR and defibrillation as soon as possible is likely to improve a return of spontaneous circulation (ROSC) and survival (8). In Taif city, as in many areas of the Kingdom of Saudi Arabia, the AHA certified course is mandatory for all primary health care physicians. These 5-hour, video based, paid courses are facilitated by AHA faculty and instructors. Every attended healthcare worker should be able to perform the skills after watching the video and the demonstration by the instructors. The practical skill of each attendant is assessed using a skill performance checklist and knowledge by a multiple-choice question (MCQ)-based written exam at the end of the course. The BLS provider card issued to successful candidates is valid for only 2 years. Worldwide, few studies have been carried out regarding assessment of knowledge and attitude of primary care physicians towards BLS and its training, despite the importance of BLS training in their clinical practice. Therefore, this study was conducted to evaluate the knowledge of primary health care physicians in Taif regarding concepts and essential elements of BLS and their attitude towards BLS training.

In the present study, although all primary health care physicians attended BLS training courses and the vast majority of them (98.9\%) had a valid BLS certificate, only $56.3 \%$ of them have satisfactory knowledge regarding BLS. This obvious discrepancy between receiving training and a valid BLS certificate from one side and being knowledgeable could be attributed to the prolonged duration between receiving the training and obtaining the certificate. Similar findings were observed in previous studies. In the USA, the CPR skills and knowledge of 160 staff members at a large teaching hospital were assessed by a multiple-choice questionnaire and a practical application, and revealed that only $32.5 \%$ of physicians passed the practical test. It was concluded that training in resuscitation may have 
improved the BLS skills and knowledge, but there is clearly a need for continued assessment of resuscitation skills of physicians (9). In a study carried out in Singapore (2009) among general practitioners (10), more than half of the physicians reported that they knew how to operate an automated external defibrillator (AED). In Nigeria (11), only $40 \%$ of physicians had attended a BLS training course, $30 \%$ knew how to operate an AED and $70 \%$ knew the meaning of AED. In Jamaica (12), knowledge of CPR guidelines was inadequate with a median score of 4 out of a possible $8.86 \%$ of all physicians who had been trained in BLS, and only $46 \%$ were certified at the time of the study. In Australia, knowledge among physicians regarding BLS and CPR for children and adults, based on Australian Resuscitation Council Guidelines was overall inadequate (13). Nambiar et al. (15) in India reported that the mean score of BLS knowledge among physicians was $8.6 \pm 3.4$ out of a maximum of 20 and $51.4 \%$ of them scored $\leq 50 \%$.

In the present study, all primary health care physicians thought that BLS training should be mandatory, and the majority of them were interested in gaining more skills and knowledge of BLS, and willing to gain additional BLS knowledge in the form of a BLS course. Overall, $65.5 \%$ of them expressed positive attitude towards BLS training. The same has been reported in a study carried out in India (14) where $77.2 \%$ of physicians suggested that BLS be included in the academic curriculum. In Singapore, Ong et al. observed that $38 \%$ of general practitioners had attended AED training and 36\% were willing to perform mouth-to-mouth ventilation during LBS (10). Abolfotouh et al. (15) observed that attending additional BLS courses for physicians who previously received BLS training improved their attitude towards BLS-AED training and observed that the number of previous BLS training courses and previous exposure to cardiac-arrest cases were significantly associated with positive attitude.

In this study, non-Saudi physicians expressed higher knowledge concerning BLS and more positive attitude towards its training than Saudi physicians did. Also, Family Medicine certified physicians had lower BLS knowledge and higher negative attitude towards its training. Both factors are closely related together as almost all Family Medicine certified physicians are Saudis. This finding could be explained by the fact that the FM residency training program does not include BLS training and having a valid BLS certificate is not mandatory for this group. Similarly, in a study conducted in the UK, it was found that the resuscitation skills and knowledge of a sample of MRCP under graduation physicians were unsatisfactory. (16)

In the present study, physicians in the age group 31-40 years were more knowledgeable regarding BLS in bivariate analysis, however, in multivariate analysis, physicians' age was not significantly associated with BLS knowledge. In Singapore, general practitioners younger than 50 years were more knowledgeable of BLS than older physicians (10). In Nigeria (11), male physicians and those aged between 30 and 40 years were more knowledgeable regarding AED operation. In India (14), mean BLS knowledge score decreased with age, and physicians with $<5$ years' experience had significantly higher scores. In the present study, also, more experienced physicians were less knowledgeable regarding BLS than less experienced physicians were. The same had been reported in Jamaica (12) where physician seniority was inversely related to knowledge scores. This could be attributed to the fact that the knowledge and skills regarding BLS is related to training regardless of the experience in work at primary health care centers, and also related to the qualification and the type of didactic teaching. Younger and less experienced physicians in PHC practice are usually more subjected to training and continuous education than older and more experienced physicians are. In Singapore (10), attending BLS training courses impacted positively on the attitude of general practitioners to perform mouth-to-mouth ventilation. In the present study, all physicians attended BLS training courses; therefore, we could not compare the impact of attending such courses on BLS knowledge. Resuscitation skills need to be refreshed after some time, and short courses can be offered to personnel who have already taken a course previously to assure effective revision. As found by Cooper et al., there was significant improvement in the knowledge and skills of people who have taken BLS courses six months after taking a short ILS (Immediate Life Support) course (17). However, in the current study, we could not prove this fact because we did not inquire about the length of time since the last BLS course.

Some studies revealed that physicians who had a valid BLS certificate had significantly more satisfactory knowledge about BLS compared to those who did not have a valid BLS certificate $(18,19)$. In the current study, only two physicians did not have a valid BLS certificate and both expressed unsatisfactory knowledge. The impact of training on knowledge about BLS was also studied in the UK among undergraduates who had been subjected to undergraduate training courses concerning BLS, and it was observed that there were major variations in BLS knowledge following the training courses (20). In the present study, physicians who had positive attitude regarding BLS training expressed less satisfactory knowledge regarding BLS. This could be explained by the fact that 
physicians who had satisfactory knowledge reported less desire and motivation to attend BLS training courses and vice versa (21).

\section{Study limitations and strengths}

Regarding the strengths, we can say that the study included sufficient representative sample size, and evaluations were done based on the recent American Heart Association guidelines. In addition, the study targeted the primary healthcare physicians, which makes it a unique study, as most of the previous studies were hospital-based. Finally, using multivariate logistic regression analysis and also adjusting the odds ratios were other positive points towards the validity and the reliability of the findings. Regarding the study limitations, we should say that only theoretical knowledge was evaluated while practical skills to perform BLS were not evaluated.

\section{Conclusions}

The present study has shown that more than half of the PHC physicians had satisfactory knowledge, although all physicians have attended BLS courses and the vast majority of them had valid BLS certificates. Saudi physicians, those having more experience in practice and FM resident physicians were less knowledgeable than their counterparts were. Attitude of the physicians towards BLS was positive in general, and Saudi physicians and family medicine residents had more negative attitude towards BLS than their counterparts had. Since the BLS certificate is validated for two years, additional courses could be given to primary health care physicians between the mandatory courses. BLS and other resuscitation skills should be a part of the FM residency program curriculum and students should be required to master the skills during their studies.

\section{Acknowledgments:}

Special thanks go to all responsible for primary health care and the Ministry of Health in Taif for their support during study preparation. Thanks and appreciation to all physicians who participated in this study for their cooperation.

\section{Conflict of Interest:}

There is no conflict of interest to be declared.

\section{Authors' contributions:}

All authors contributed to this project and article equally. All authors read and approved the final manuscript.

\section{References:}

1) ECC Committee, Subcommittees and Task Forces of the American Heart Association. 2005 American Heart Association Guidelines for Cardiopulmonary Resuscitation and Emergency Cardiovascular Care. Circulation. 2005; 112(24 Suppl): IV1-203.

2) Seraj MA, Harvey PJ. 15 years of experience with cardiopulmonary resuscitation in the Kingdom of Saudi Arabia: a critical analysis. Prehosp Disaster Med. 1999; 14(3): 180-5. doi: 10.1017/S1049023X00027564. PMID: 10724743.

3) Berg RA, Hemphill R, Abella BS, Aufderheide TP, Cave DM, Hazinski MF, et al. Part 5: adult basic life support: 2010 American Heart Association Guidelines for Cardiopulmonary Resuscitation and Emergency Cardiovascular Care. Circulation. 2010; 122(18 Suppl 3): S685-705. doi: 10.1161/CIRCULATIONAHA.110.970939. PMID: 20956221.

4) Kingdom of Saudi Arabia. Taif Governorate census. Available from: http://www.taifcity.gov.sa

5) Online Sample Siza Calculator. Available from: https://www.surveysystem.com/sscalc.htm

6) Neumar RW, Shuster M, Callaway CW, Gent LM, Atkins DL, Bhanji F, et al. Part 1: Executive summary 2015 American Heart Association guidelines update for cardiopulmonary resuscitation and emergency cardiovascular care. Circulation. 2015; 132: S315-67. doi: 10.1161/CIR.0000000000000252. PMID: 26472989.

7) Kanstada BK, Nilsena SA, Fredriksen K. CPR knowledge and attitude to performing bystander CPR among secondary school students in Norway. Resuscitation. 2011; 82(8): 1053-9. doi: 10.1016/j.resuscitation.2011.03.033. PMID: 21531067.

8) Travers AH, Perkins GD, Berg RA, Castren M, Considine J, Escalante R, et al. Part 3: Adult Basic Life Support and Automated External Defibrillation 2015 International Consensus on Cardiopulmonary Resuscitation and Emergency Cardiovascular Care Science With Treatment Recommendations. Circulation. 2015; 132(16 suppl 1): S51-83. doi: 10.1161/CIR.0000000000000272. PMID: 26472859. 
9) Goucke CR, Dobb GJ. Cardiopulmonary resuscitation skills of hospital medical and nursing staff members. Med J Aust. 1996; 145(10): 496-7.

10) Ong ME, Yap S, Chan KP, Sultana P, Anantharaman V. Knowledge and attitudes towards cardiopulmonary resuscitation and defibrillation amongst Asian primary health care physicians. Open Access Emerg Med. 2009; 1: 11-20. PMID: 27147830, PMCID: PMC4806819.

11) Olajumoke TO, Afolayan JM, Raji SA, Adekunle MA. Cardiopulmonary resuscitation-knowledge, attitude $\&$ practices in Osun State, Nigeria. J West Afr Coll Surg. 2012; 2(2): 23-32. PMID: 27182506. PMCID: PMC4767296.

12) Howell P, Tennant I, Augier R, Gordon-Strachan G, Harding-Goldson H. Physicians' knowledge of cardiopulmonary resuscitation guidelines and current certification status at the University Hospital of the West Indies, Jamaica. West Indian Med J. 2014; 63(7): 739-43. PMID: 25867559.

13) Kila T, Yockopua S. Knowledge of cardiopulmonary resuscitation among doctors at the Port Moresby General Hospital. P N G Med J. 2012; 55(1-4): 76-87. PMID: 25338477.

14) Nambiar M, Nedungalaparambil NM, Aslesh OP. Is current training in basic and advanced cardiac life support (BLS \& ACLS) effective? A study of BLS \& ACLS knowledge amongst healthcare professionals of North-Kerala. World J Emerg Med. 2016; 7(4): 263-9. doi: 10.5847/wjem.j.1920-8642.2016.04.004. PMID: 27942342, PMCID: PMC5143309.

15) Abolfotouh MA, Alnasser MA, Berhanu AN, Al-Turaif DA, Alfayez AI. Impact of basic life-support training on the attitudes of health-care workers toward cardiopulmonary resuscitation and defibrillation. BMC Health Serv Res. 2017; 17(1): 674. doi: 10.1186/s12913-017-2621-5. PMID: 28938914, PMCID: PMC5610457.

16) Chin D, Morphet J, Coady E, Davidson C. Assessment of cardiopulmonary resuscitation in the membership examination of the Royal College of Physicians. J R Coll Physicians Lond. 1997; 31(2): 198-201. PMID: 9131523.

17) Cooper S, Johnston E, Priscott D. Immediate life support (ILS) training impact in a primary care setting? Resuscitation. 2007; 72: 92-9. doi: 10.1016/j.resuscitation.2006.06.004. PMID: 17069948.

18) Alkabsh M. Knowledge and attitude of primary health care providers towards Basic Life Support (BLS) in Jeddah Governorate, 2006. A Dissertation submitted to a partial fulfillment of the requirement for the Saudi Board of Family Medicine, 2006.

19) Akpek EA, Kayhan Z. Knowledge of basic life support: a pilot study of the Turkish population by Baskent University in Ankara. Resuscitation. 2003; 58(2): 187-92. doi: 10.1016/S0300-9572(03)00126-6.

20) Jordan T, Bradley P. A survey of basic life support training in various undergraduate health care professions. Resuscitation. 2002; 47(3): 321-3. doi: 10.1016/S0300-9572(00)00308-7.

21) Chaudhari MS, Panchal NN, Kamat HV, Ganjiwale J. Knowledge of 2015 basic life support (BLS) guidelines among doctors and nursing staff of a rural based tertiary care hospital, in western India: Current status and requirement. Indian Journal of Clinical Anaesthesia. 2017; 4(2): 193-7. 